\title{
Design of a Highly Efficient Subwavelength Antireflective Structure for Solar Cells
}

\author{
Lin Chen $\mathbb{D}^{1,2}$ and Zhao Huang $\mathbb{D}^{2}$ \\ ${ }^{1}$ MOE Key Laboratory of Material Physics and Chemistry Under Extraordinary Conditions and Shaanxi \\ Key Laboratory of Optical Information Technology, School of Physical Science and Technology, \\ Northwestern Polytechnical University, Xi'an 710129, China \\ ${ }^{2}$ AVIC Xi'an Flight Automatic Control Research Institute, Xi'an 710065, China \\ Correspondence should be addressed to Lin Chen; chenlin618@outlook.com
}

Received 5 March 2021; Revised 9 January 2022; Accepted 12 January 2022; Published 7 February 2022

Academic Editor: Rujiang Li

Copyright $\odot 2022$ Lin Chen and Zhao Huang. This is an open access article distributed under the Creative Commons Attribution License, which permits unrestricted use, distribution, and reproduction in any medium, provided the original work is properly cited.

\begin{abstract}
An efficient optical antireflective (AR) structure plays a vital role in high-performance thin-film solar cells. Here, we design a surface relief AR structure consisting of a two-dimensional (2D) array of a subwavelength ring and pillar-shaped feature, capable of suppressing optical reflection over a wide spectral window of the solar spectrum. Our simulations show that the weighted average reflectance of the subwavelength AR structure is as low as $4.2 \%$ in the $400-1100 \mathrm{~nm}$ spectral range in the normal incidence condition and almost 10 -fold reduction compared with a bare silicon surface. When placed on the front side of a simple Si thinfilm photovoltaic solar cell, this subwavelength AR structure leads to an improved light absorption with simulated results showing an increase of $53 \%$ short-circuit current compared to a flat solar cell. Besides, our simulations show that this AR structure could, in principle, perform well against reasonable fabrication errors.
\end{abstract}

\section{Introduction}

With the degradation of the environment and climate change on earth, renewable energy has become a key theme in sustainable social development. In the past decades, photovoltaic technology has attracted much attention for its potential to provide renewable energy instead of traditional fossil fuels [1-3]. While there is a growing trend of this technology being deployed in industrial environments, its cost is still one of the main factors limiting worldwide civil end use [4]. One potential way to reduce the cost is to increase photovoltaic solar cells' efficiency by reducing the reflection of solar cells and increasing their absorption $[5,6]$. In this research direction, several methods are commonly used for reducing the unwanted Fresnel reflection on the surface between two adjacent materials with different refractive indices, such as single or multiple-layer antireflective (AR) coatings [7, 8] and light-trapping nanostructures [9]. It is well-known that a single $\lambda / 4$ thick dielectric layer will significantly reduce Fresnel reflection by realizing destructive interference at the reflection path over a narrow spectral bandwidth centered at $\lambda$. Although applying multiple layers can broaden the antireflection spectral window [10], it is difficult to find proper material combinations and grow perfect multilayers at a low cost. Alternatively, moth-eye structure and regular or random pyramids can be used in solar cells to improve light absorption efficiency $[11,12]$ because they increase effective optical scattering paths to allow more photons to be absorbed. They are often used in wafer-based silicon solar cells, which have a typical active layer thickness on the order of $10 \mu \mathrm{m}$. However, typical thinfilm silicon solar cells only have a thickness of about $3 \mu \mathrm{m}$. As a result, these light-trapping structures are not suitable [13]. Because of their tailored optical responses, subwavelength structures have attracted much attention in designing antireflective surfaces. For example, Spinelli et al. presented a new concept to suppress the reflection of light from a silicon surface in the $450-900 \mathrm{~nm}$ spectral range [14] using a very 
simple nanostructure. Along this direction, there have been many proposed and demonstrated outstanding absorption enhancing nanostructures, for instance, tungsten elliptical arrays on monolayer molybdenum disulfide, trapezoid-pyramidal structure-based PEDOT: PSS/c-Ge, Ag nanoparticles, and $\mathrm{TiO}_{2}$-inverted triangular prism, $\mathrm{Ti}$ ring on $\mathrm{SiO}_{2}-\mathrm{Si}_{3} \mathrm{~N}_{4}$-Ti thin films [15-19]. Notice that these nanostructures all require heterogeneous integration of exterior materials, complicating the device fabrication processes and potentially defeats the purpose of lowering the solar cells' cost. A straightforward solution to bypass these issues could be surface engineering the solar cell by directly patterning the thin-film silicon layer.

In this study, a broadband surface relief subwavelength AR structure for thin-film silicon solar cells is designed and optimized. It consists of a two-dimensional (2D) array of rings and pillars. Its weighted average reflectance is as low as $4.2 \%$ over the $400-1100 \mathrm{~nm}$ spectral range, which is almost an order of magnitude reduction compared with a bare silicon surface. The directivity of the antireflection and polarization sensitivity of this structure are further studied in detail. Besides, our investigation on the light absorption property of a $3 \mu \mathrm{m}$ thick thin-film silicon solar cell suggests that the generated short-circuit current of a simple but representative solar cell can be improved by more than $50 \%$. Finally, the fabrication tolerance analysis shows that our subwavelength AR structure can have geometric tolerance to a certain extend.

\section{Results and Discussion}

Figure 1 shows the schematic view and the section view of the proposed subwavelength AR structure. It is a periodic structure that can be integrated onto the surface of the top silicon layer of solar cells. Its unit cell consists of an outer ring and a middle pillar. The outer and the inner diameters of the ring structure are $d_{\text {out }}$ and $d_{\text {in }}$, respectively. The diameter of the pillar is $d_{\text {core }}$. The outer ring and the middle pillar have the same height, $h$. Moreover, the periods in the $x$ and $y$ directions are the same and are fixed at $p=500 \mathrm{~nm}$.

To minimize the reflection over a wide wavelength range, it is crucial to find the optimum geometric parameters of the subwavelength AR structure. We use the averaged reflectance, $R_{\text {ave }}$, weighted with the AM1.5 solar spectrum in the $400-1100 \mathrm{~nm}$ spectral range, as a quantitative figure of metric for the optimization design. It is defined as follows:

$$
R_{\mathrm{ave}}=\frac{\int_{400}^{1100} R(\lambda) \times \Phi_{\mathrm{AM} 1.5} \mathrm{~d} \lambda}{\int_{400}^{1100} \Phi_{\mathrm{AM} 1.5} \mathrm{~d} \lambda},
$$

where $\lambda$ is the wavelength of the incident light, $R(\lambda)$ is the corresponding wavelength-dependent reflectance of the front surface of solar cells, and $400 \mathrm{~nm}$ and $1100 \mathrm{~nm}$ are the lower and upper boundaries of the absorption wavelength of interest for the silicon solar cells. The electromagnetic response of this structure is simulated using commercially available finite-difference time-domain (FDTD) tools. A simulation domain of $0.5 \times 0.5 \times 1 \mu \mathrm{m}(x \times y \times z)$ is used with periodic boundary conditions in the $x$ and $y$ directions and a perfectly matched layer condition in the $z$-direction. The light source is a broadband (400-1100 nm) plane wave with a varying angle of incidence to the substrate. After checking the numerical convergence, the simulation mesh grid is set to $10 \mathrm{~nm}$ over the entire simulation domain, with a refined override setting of $5 \mathrm{~nm}$ over the region occupied by the subwavelength ring and pillar. The optical dispersion property of silicon is taken into account in all numerical simulations [20]. Our investigation using particle swarm optimization yields the following main geometric parameters of the optimized subwavelength AR structure, as given in Table 1. The scattering cross-section of its unit is analyzed [14], and the corresponding result is shown in Figure 1(c). It suggests that this structure has a greatly enhanced normalized scattering cross-section across the whole spectrum of interest (400-1100 nm). The peak of the scattering cross-section is about ten times the geometric cross-section at the wavelength of $655 \mathrm{~nm}$, which is near the peak wavelength of the solar spectrum. Also, from the inset of Figure 1(c), there is a strong leaky channel into the silicon substrate. These factors are mainly responsible for the reduced reflection and enhanced absorption in the photovoltaic solar cell.

Figure 2(a) shows the simulated reflectance spectra of a silicon substrate with our optimized AR structure under normal incidence. The result suggests that the reflectance varies only slightly with the wavelength, and for the entire spectrum from $400 \mathrm{~nm}$ to $1100 \mathrm{~nm}$, it is well below $10 \%$. The reflectance from a bare flat silicon substrate is also simulated and shown as the black curve for comparison. It has a minimum value of $30 \%$, far exceeding that of our subwavelength structure. According to equation (1), the weight reflectance, $R_{\text {ave }}$, over the entire spectral window for our case is less than $4 \%$. It strongly indicates that this kind of surface structure is beneficial in suppressing optical reflection. The trend shown here agrees well with reports elsewhere [21].

For the photovoltaic application, an AR optical structure must show low reflectance over a wide range of incident angles for both polarizations. Figure 2(b) shows the weighted averaged reflectance $R_{\text {ave }}$ simulated for s-polarized (black curve with solid square symbols) and p-polarized (red curve with solid dot symbols) light over the 400-1100 nm spectral window as a function of the angle of incidence (AOI) from $10^{\circ}$ to $60^{\circ}$. Overall, the reflectance for $\mathrm{s}$ and $\mathrm{p}$-polarizations increases gradually with the angle of incidence. The reflectance, however, remains below $10 \%$ for both s and p-polarizations for the incident angle up to $30^{\circ}$. Moreover, the averaged reflectivity for p-polarization is slightly larger than that for s-polarization at large angles suggesting our subwavelength structure shows a mild polarization dependence for $\mathrm{s}$ and p-polarizations.

To examine our subwavelength AR structure's efficacy, a simple three-layer $\mathrm{Si}$ thin-film solar cell is modeled. It consists of an active layer of a $3 \mu \mathrm{m}$ thickness c-Si, sandwiched by the subwavelength AR structure on the top and a $0.5 \mu \mathrm{m} \mathrm{Al} \mathrm{slab}$ at the bottom. The absorbed optical energy is first calculated to study the photocurrent in the solar cell. The absorbed energy density per unit volume of the active layer, $P_{a b s}$, could be derived from the following formula: 


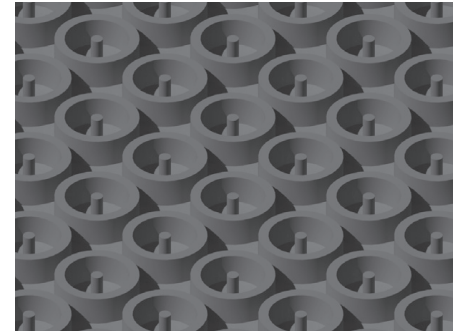

(a)

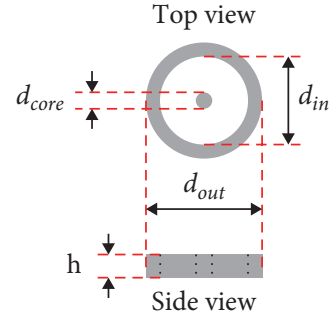

(b)

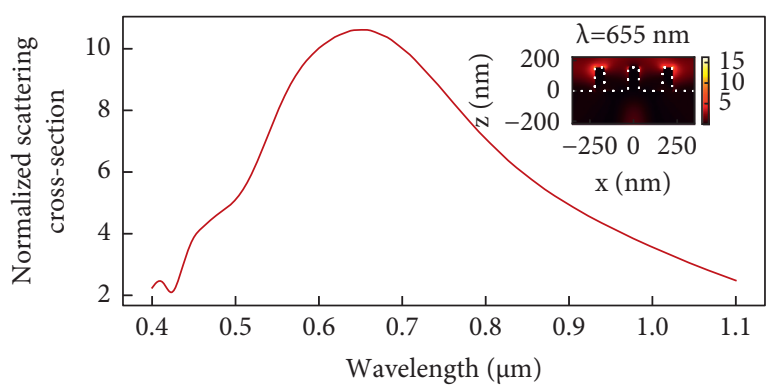

(c)

FIgURE 1: (a) Schematic view of the silicon subwavelength AR structure used in the simulations. (b) Main geometric parameters of the silicon subwavelength AR structure. (c) Normalized scattering cross-section (inset: normalized intensity profile at the resonance wavelength).

TABLE 1: The main geometric parameters of the subwavelength AR structure $(\mu \mathrm{m})$.

\begin{tabular}{lcccc}
\hline$d_{\text {core }}$ & $d_{\text {in }}$ & $d_{\text {out }}$ & $h$ & \\
0.06 & 0.333 & 0.44 & 0.155 & 0.5 \\
\hline
\end{tabular}

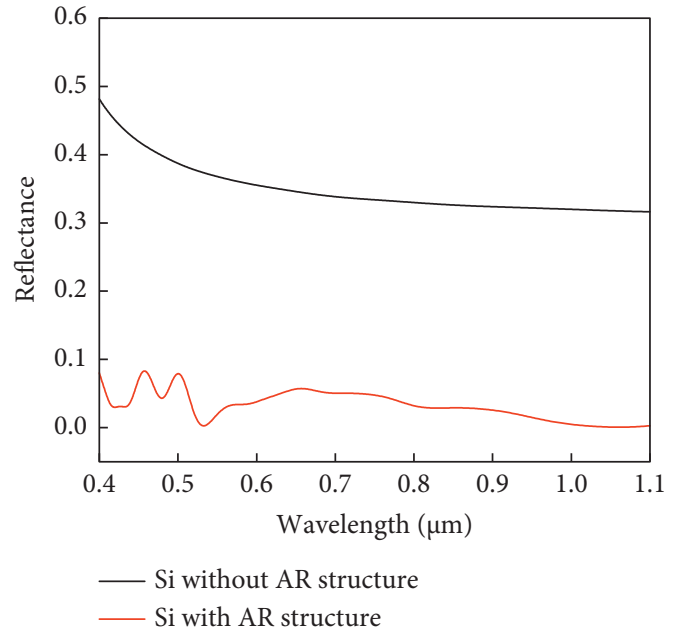

(a)

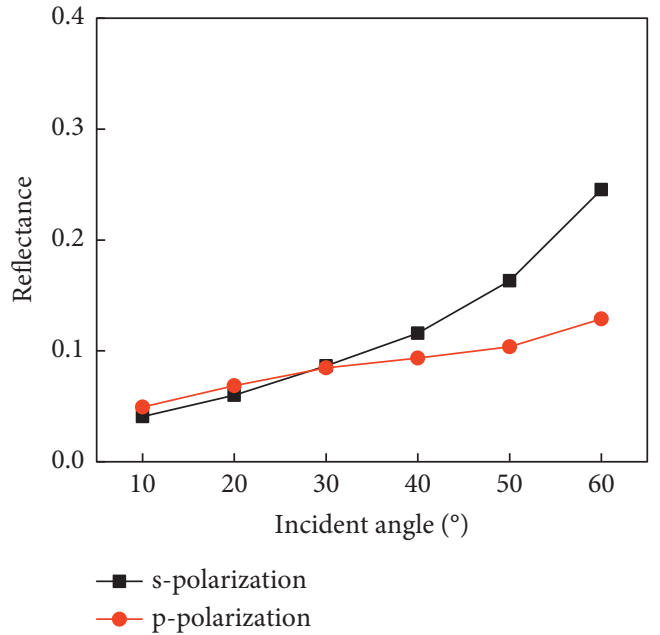

(b)

FIGURE 2: (a) Reflectance spectra of the silicon substrate with and without AR subwavelength structures under normal incidence. (b) Reflectance for s-polarized (black curve with solid square symbols) and p-polarized (red curve with solid dot symbols) incident beam over the angle of incidence (AOI) from $10^{\circ}$ to $60^{\circ}$.

$$
P_{a b s}=-0.5 \omega|\vec{E}|^{2} \operatorname{imag}(\varepsilon),
$$

where $\omega$ is the incident light's angular frequency, $\vec{E}$ is the electric field, and $\operatorname{imag}(\varepsilon)$ is the imaginary part of the permittivity. Figure 3 shows the calculated results of the spatial distribution of $P_{a b s}$ at the wavelength of $600 \mathrm{~nm}$ and $1100 \mathrm{~nm}$, respectively. In the first case, the absorption occurs strongly in the whole $\mathrm{Si}$ active layer underneath the subwavelength AR structure. In the latter case, the spatial absorption is much lower in the Si active layer. These results agree well with the absorption spectra above. Besides, we also see an interference pattern due to backward reflections at the interfaces, thereby increasing the effective optical path and light absorption.
To roughly estimate the enhancement of photocurrent with our proposed AR structures, we assume an ideal extraction of charged carriers, and all electron-hole pairs contribute to the photocurrent in both cases with and without AR structures. The short-circuit current density $J_{s c}$ under the standard solar irradiance can be calculated according to reference [22], using the following two equations:

$$
\begin{aligned}
\mathrm{QE}(\lambda) & =\frac{P_{a b s}(\lambda)}{P_{\text {in }}(\lambda)}, \\
J_{s c} & =e \int \frac{\lambda}{h c} \mathrm{QE}(\lambda) I_{\mathrm{AM} 1.5}(\lambda) \mathrm{d}(\lambda),
\end{aligned}
$$




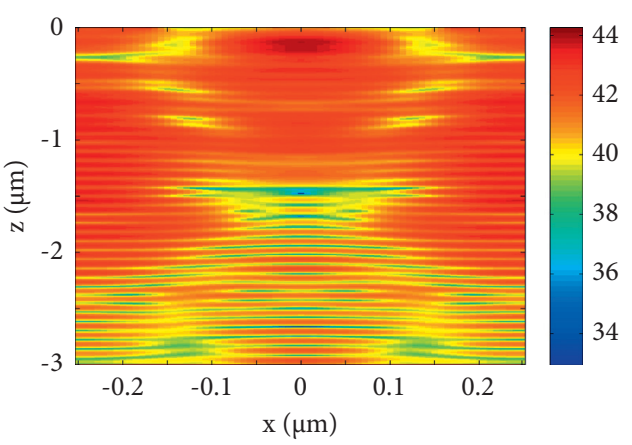

(a)

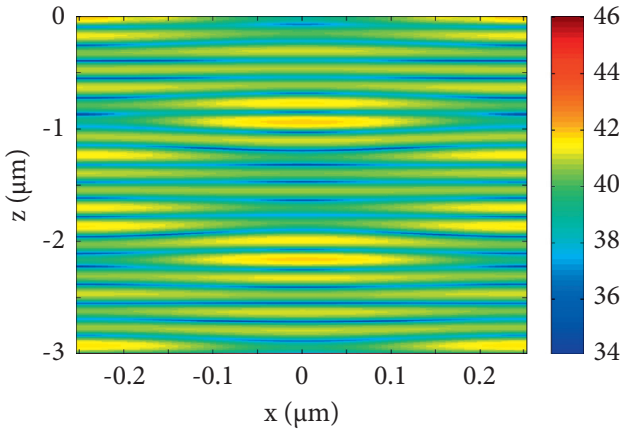

(b)

FIgURE 3: The spatial absorption profiles at $600 \mathrm{~nm}$ (a) and $1100 \mathrm{~nm}$ (b).

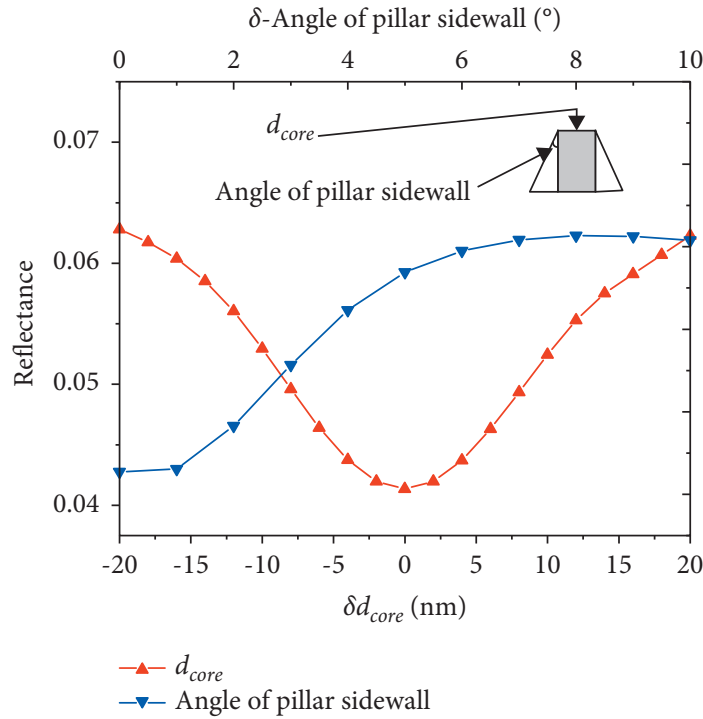

(a)

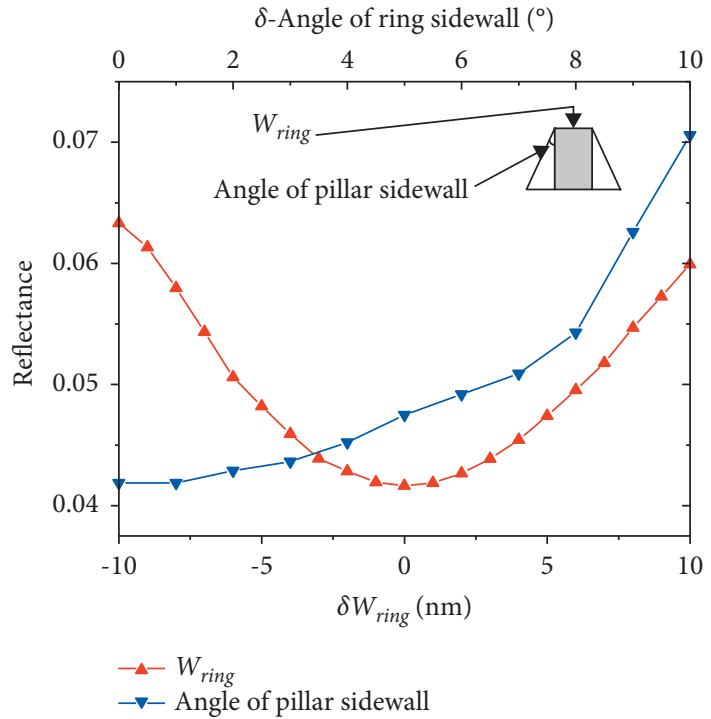

(b)

FIgURE 4: Effect of fabrication variations of the middle pillar (a) and outer ring (b) on the reflectance.

where $e$ is the charge of an electron, $h$ is Plank's constant, $c$ is the speed of light in the free space, and $I_{\mathrm{AM} 1.5}(\lambda)$ is the AM 1.5 solar spectrum. Our calculation shows that $J_{s c}$ is around $28.6 \mathrm{~mA} / \mathrm{cm}^{2}$ with a $53 \%$ increment compared to a device without an AR layer $\left(18.7 \mathrm{~mA} / \mathrm{cm}^{2}\right)$.

Fabrication tolerance plays a vital role in the performance of the subwavelength structures. To study the fabrication tolerance of the proposed subwavelength AR structure, the performance of $\mathrm{Si}$ subwavelength $\mathrm{AR}$ structure under different degrees of size deviation is simulated. Fabrication variations of the AR structure's outer ring and middle pillar are considered. Especially, the impact of the deviation of their diameters and the sidewall angles from the optimal values is studied. The simulated results for weighted reflectance with the AM1.5 solar spectrum are shown in Figures 4(a) and 4(b), respectively. Noticeably, the results show very slight degradation of the reflectance within the variation range of $\pm 10 \mathrm{~nm}$ and $0-10^{\circ}$, respectively. Therefore, our proposed AR structures could in principle perform well against minor fabrication errors.

\section{Conclusion}

We have proposed a unique subwavelength structure composed of pillars and rings that can largely suppress the $\mathrm{Si}$ surface's reflection across a broadband wavelength range. Our simulations show that the averaged reflectance, weighted with the AM1.5 solar spectrum in the $400-1100 \mathrm{~nm}$ spectral range, can be reduced from $35 \%$ down to only $4.2 \%$. Notably, the modeled short-circuit current for a simple thinfilm solar cell exhibits a $53 \%$ increment compared to that of a device without the AR layer. Besides, we show that the subwavelength AR structures are robust to certain degrees of fabrication errors. The results demonstrated here provide a straightforward and low-cost method, feasible with the current nanoimprinting technology, for fully exploring the advantages of thin-film solar cells.

\section{Data Availability}

The research data used to support the findings of this study are available from the corresponding author upon request. 


\section{Conflicts of Interest}

The authors declare that they have no conflicts of interest.

\section{References}

[1] J. Nelson, The Physics of Solar Cells, pp. 211-251, Imperial College Press, London, 2008.

[2] Y. Hamakawa, Thin-film Solar Cells: Next Generation Photovoltaics and its Applications, pp. 15-39, Springer, Berlin, 1980.

[3] M. A. Green, "The path to $25 \%$ silicon solar cell efficiency: history of silicon cell evolution," Progress in Photovoltaics: Research and Applications, vol. 17, pp. 183-189, 2009.

[4] A. G. Aberle, "Surface passivation of crystalline silicon solar cells: a review," Progress in Photovoltaics: Research and Applications, vol. 8, pp. 473-487, 2000.

[5] Y. Liu and O. J. Guy, "Refractive index graded anti-reflection coating for solar cells based on low cost reclaimed silicon," Microelectronic Engineering, vol. 110, pp. 418-421, 2013.

[6] B. Hussain and A. Ebong, "Specifications of $\mathrm{ZnO}$ growth for heterostructure solar cell and PC1D based simulations," Solar Energy Materials and Solar Energy Cells, vol. 5, pp. 516-521, 2015.

[7] Y. Xu, B. Zhang, W. H. Fan, D. Wu, and Y. Han, "Sol-gel broadband anti-reflective single-layer silica films with high laser damage threshold," Thin Solid Films, vol. 440, pp. 180183, 2003.

[8] S. E. Lee, S. W. Choi, and J. Yi, "Double-layer anti-reflection coating using $\mathrm{MgF}_{2}$ and $\mathrm{CeO}_{2}$ films on a crystalline silicon substrate," Thin Solid Films, vol. 376, pp. 208-213, 2000.

[9] J. Van de Groep, P. Spinelli, and A. Polman, "Single-step softimprinted large-area nanopatterned antireflection coating," Nano Letters, vol. 15, pp. 4223-4228, 2015.

[10] M. F. Schubert, F. W. Mont, S. Chhajed, D. J. Poxson, J. K. Kim, and E. F. Schubert, "Design of multilayer antireflection coatings made from co-sputtered and low-refractiveindex materials by genetic algorithm," Optics Express, vol. 16, pp. 5290-5298, 2008.

[11] C. Sun, P. Jiang, and B. Jiang, "Broadband moth-eye antireflection coatings on silicon," Applied Physics Letters, vol. 92, Article ID 061112, 2008.

[12] A. Deinega, I. Valuev, B. Potapkin, and Y. Lozovik, "Minimizing light reflection from dielectric textured surfaces," Journal of the Optical Society of America. A, vol. 28, pp. 770-777, 2011.

[13] M. L. Brongersma, Y. Cui, and S. Fan, "Light management for photovoltaics using high-index nanostructures," Nature Materials, vol. 13, pp. 451-460, 2014.

[14] P. Spinelli, "Broadband omnidirectional antireflection coating based on subwavelength surface Mie resonators," Nature Communications, vol. 3692 pages, 2012.

[15] J. Li, X. Chen, Z. Yi et al., "Broadband solar energy absorber based on monolayer molybdenum disulfide using tungsten elliptical arrays," Materials Today Energy, vol. 16, Article ID 100390, 2020

[16] F. Zhao, X. Chen, Z. Yi et al., "Study on the solar energy absorption of hybrid solar cells with trapezoid-pyramidal structure based PEDOT: PSS/c-Ge," Solar Energy, vol. 204, pp. 635-643, 2020.

[17] F. Zhao, Y. Yi, J. Lin et al., "The better photoelectric performance of thin-film $\mathrm{TiO} 2 / \mathrm{c}-\mathrm{Si}$ heterojunction solar cells based on surface plasmon resonance," Results in Physics, vol. 28, Article ID 104628, 2021.
[18] F. Zhou, F. Qin, Z. Yi et al., "Ultra-wideband and wide-angle perfect solar energy absorber based on Ti nanorings surface plasmon resonance," Physical Chemistry Chemical Physics, vol. 23, pp. 17041-17048, 2021.

[19] J. Su, H. Yang, Y. Xu et al., "Based on ultrathin PEDOT: PSS/cGe solar cells design and their photoelectric performance," Coatings, vol. 11, no. 7, p. 748, 2021.

[20] E. D. Palik, Handbook of Optical Constant of Solids I, Academic Press, San Diego, CA, USA, 1985.

[21] Y. J. Hung, S. Lee, K. Wu, Y. Tai, Y. Tai, and Y. Pan, “Antireflective silicon surface with vertical-aligned silicon nanowires realized by simple wet chemical etching processes," Optics Express, vol. 19, pp. 15792-15802, 2011.

[22] X. Shen, Q. Wang, and P. Wangyang, "Broadband antireflection and light trapping in thin-film silicon solar cells with hemisphere arrays," IEEE Photonics Technology Letters, vol. 28 , no. 13 , pp. $1477-1480,2016$. 